\title{
Formation of massive stars
}

\author{
Maria T. Beltrán ${ }^{1}$ \\ ${ }^{1}$ INAF, Osservatorio Astrofisico di Arcetri, \\ Largo E. Fermi 5, 50125 Firenze, Italy \\ email: mbeltran@arcetri.astro.it
}

\begin{abstract}
The formation of high-mass stars represents a challenge from both a theoretical and an observational point of view. Here, we present an overview of the current status of the observational research on this field, outlining the progress achieved in recent years on our knowledge of the initial phases of massive star formation. The fragmentation of cold, infrared-dark clouds, and the evidence for star formation activity on some of them will be discussed, together with the kinematics of the gas in hot molecular cores, which can give us insights on the mechanism leading to the birth of an OB star.
\end{abstract}

Keywords. ISM: molecules, stars: formation, radio lines: ISM

\section{Introduction}

How do massive (>8 $M_{\odot}$ ) stars form? Do they form like lower-mass stars or in a fundamentally different way? These crucial questions have profound implications for many areas of astrophysics including high-redshift Population III star formation, galaxy formation and evolution, galactic center environments and super-massive black hole formation, star and star cluster formation, and planet formation around stars in clusters.

High-mass stars are rare objects that form very fast. What is more, the closest highmass star-forming regions are located further away than the nearest low-mass ones. They form deeply embedded in clusters, where the extinction is very high and there is strong interaction with the environment and feedback from other recently formed stars. All this makes the study of the formation of massive stars challenging, due to the difficulty of tracing the primordial configuration of the molecular cloud, and the impossibility of studying the earliest stages of massive star-formation at optical or even infrared wavelengths.

From a theoretical point of view, massive stars represent a challenge because of their shorter evolutionary timescales. For massive protostars, the Kelvin-Helmholtz timescale is shorter than the free-fall timescale. Therefore, massive stars reach the Zero Age Main Sequence still deeply embedded in the parental cloud and with ongoing accretion. According to theoretical predictions, at this stage, their powerful radiation pressure appears to be strong enough to halt the infalling material (Kahn 1974; Wolfire \& Cassinelli 1987), which should inhibit further growth of the star beyond the limit of $8 M_{\odot}$ (although recent 3-D radiation hydrodynamic simulations by Krumholz et al. (2009) indicate that radiation pressure may not be a problem). In order to solve this problem, competitive accretion has been proposed (Bonnell \& Bate 2002), that predicts that initially a molecular cloud fragments in low-mass cores of a Jeans mass $\sim 0.5-1 M_{\odot}$, which form stars that compete to accrete mass from the common gas reservoir. Protostars located near the center of the gravitational potential well accrete at a higher accretion rate because of a stronger gravitational pull. Core accretion (McKee \& Tan 2002) may also be a viable theoretical solution to overcome the radiation pressure with the ram pressure of the infalling material. In this turbulent accretion model, stars form via monolithic collapse of a massive core fragmented from the natal molecular cloud. Massive star formation 


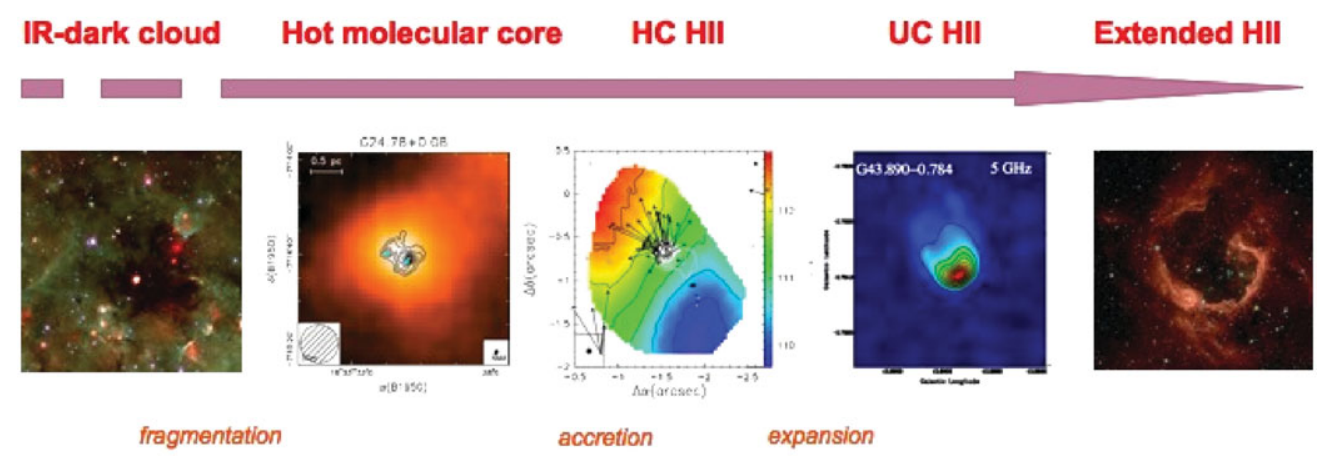

Figure 1. Evolutionary sequence for high-mass stars: from cold IRDCs to chemically rich HMCs to HiI regions.

is therefore, in this picture, a sort of scale-up version of low-mass star formation, with collapse, accretion, rotation, and outflows.

Due to the short timescales needed to form a massive star, it is very difficult to trace the earliest stages of its formation and define an evolutionary sequence for high-mass stars, similar to the one defined for low-mass star. However, a reasonable hypothesis may be that the formation process starts with the fragmentation of a cold cloud, sometimes dark at IR wavelengths (IRDC). Subsequent infall and heating inside each fragment (core) eventually leads to the formation of chemically rich hot molecular cores (HMCs), which are the cradle of high-mass stars. Accretion continues onto high-mass protostars that gain mass. The UV-radiation from the young embedded star will develop a bubble of ionized gas, known as hypercompact HıI (HC HII) region that eventually will start expanding, becoming first an ultracompact HiI (UC His) region and later on, an extended or giant HII region. Eventually, the gas is dissipated by the ionized winds, exposing the newly formed OB star (see Fig. 1).

In this contribution I will focus on the observational aspects of the earliest stages (IRDCs and HMCs) of massive star formation, which are crucial to understand how massive stars form, and I will review the observational evidence for rotation, outflows, and collapse in massive star-forming regions.

\section{Infrared dark clouds}

Massive stars form in parsec scale high-density clumps inside cold molecular clouds that when seen in absorption at IR wavelengths against the bright background are called IRDCs. These IRDCs were first detected in absorption at $8 \mu \mathrm{m}$ up to $21 \mu \mathrm{m}$ with ISO and MSX (Egan et al. 1998; Carey et al. 1998), and later on, they have been seen in absorption at $24 \mu \mathrm{m}$, sometimes up to $70 \mu \mathrm{m}$, with Spitzer (Rathborne et al. 2006), and more recently with Herschel (Peretto et al. 2010). Millimeter and submillimeter continuum observations have revealed extended cold dust emission associated with IRDCs (e.g. Schuller et al. 2009; Rosolowsky et al. 2010). These clouds have typically masses of $10^{3}-10^{4} M_{\odot}$, sizes of 1 to $5 \mathrm{pc}$, densities of $\sim 10^{5} \mathrm{~cm}^{-3}$, and temperatures lower than $20 \mathrm{~K}$.

Temperature structure. The knowledge of the initial stages of massive star formation, and in particular of IRDCs has experienced a significant step forward thanks to the advent of IR and submillimeter telescopes such as Spitzer and Herschel. In particular, the open time key project Hi-GAL (Molinari \& Hi-GAL Consortium 2010), which will observe the inner part of the Galactic Plane with Herschel in five bands, from 70 to $500 \mu \mathrm{m}$, will allow to construct well constrained spectral energy distributions (SEDs) for 
these objects, and determine their internal temperature and column density distributions. Using the data obtained during the Herschel Science Demonstration Phase, Peretto et al. (2010) have developed a simple pixel-by-pixel SED fitting method, and have recovered the spatial variations of both the dust column density and temperature within the IRDCs. Their analysis shows that IRDCs are not isothermal, but the dust temperature decreases significantly from background temperatures of $20-30 \mathrm{~K}$ to minimum temperatures of 8-15 K within the clouds. Knowing the temperature and its fluctuations is very important to understand fragmentation in these infrared-dark clouds. In fact, recent hydrodynamical simulations including radiative transfer (Bate 2009; Krumholz et al. 2010) show that protostellar heating could reduce significantly fragmentation on small scales.

Core fragmentation. Recent Submillimeter Array (SMA) high-angular resolution observations of IRDCs in the G28.34+0.06 region, have shown that IRDCs fragment into smaller cores with masses of 22 to $64 M_{\odot}$ and projected separations of $0.19 \mathrm{pc}$ (Zhang et al. 2009). Despite the fact that the SMA observations spatially resolve the Jeans length of $0.05 \mathrm{pc}$, the core masses are 10 times larger than the Jeans mass of $1 M_{\odot}$, for a temperature of $16 \mathrm{~K}$. This suggests that fragmentation may not be controlled just by thermal pressure and gravity, but that other stabilizing factors such as turbulence and maybe magnetic fields or radiative feedback are required to account for the large mass observed. In fact, the observed $\mathrm{NH}_{3}$ line width in one of the cores is a factor 8 larger than the thermal line width (Wang et al. 2008), suggesting that supersonic turbulence might play a crucial role in the core fragmentation.

Star formation activity. One of the hot topics in massive star formation is how prestellar are IRDCs. High-sensitivity Spitzer and Herschel observations have shown that many IRDCs show evidence for active star formation. Some of them show enhanced, slightly extended $4.5 \mu \mathrm{m}$ emission, called "green-fuzzies" by Chambers et al. (2009), and broad SiO line emission (Jiménez-Serra et al. 2010) which indicates shocked gas. These shocks could be a remnant of the IRDC formation process, or be produced by decelerated or recently processed gas in large-scale outflows driven either by 8 and $24 \mu \mathrm{m}$ embedded sources, or by an undetected and wide spread population of lower mass protostars. In some cores bright $8 \mu \mathrm{m}$ continuum emission, probably from HiI regions, has been detected or $3.6 \mu \mathrm{m}$ emission from unextincted stars. Finally, some cores show bright, compact $24 \mu \mathrm{m}$ emission that indicates deeply embedded protostars (Chambers et al. 2009).

Based on the evidence of active star formation, Rathborne et al. (2010) classify the IRDCs in five specific groups: quiescent, intermediate, active, red, and blue cores. Quiescent cores show total absence of star formation, with no significant 3-8 $\mu \mathrm{m}$ nor $24 \mu \mathrm{m}$ emission. Intermediate cores contain either a "green-fuzzy" or a $24 \mu \mathrm{m}$ point source, but not both. Active cores contain a "green-fuzzy" and a $24 \mu \mathrm{m}$ point source, and red and blue cores are associated with bright 8 and $3.6 \mu \mathrm{m}$ emission, respectively. The dust temperatures and luminosities are higher for cores with active, high-mass star formation than for quiescent cores, as expected if the evolutionary sequence described in Fig. 1 is valid and the quiescent cores have no internal heating source to significantly heat the dust. On the other hand, the mass histograms of active and quiescent cores are similar, which suggests that the associated stellar populations are nearly the same, and that the differences in their temperatures and luminosities are due to different evolutionary stages. In this scenario, the cooler quiescent cores may be the pre-stellar precursors of the warmer and more active protostellar cores. The evolutionary differences between the groups are also seen in the bolometric luminosity versus core dust mass diagram (see Fig. 10 of Rathborne et al. 2010), where for a given mass, the luminosity increases from the quiescent to the active cores as a result of an increase of the protostellar activity. 
Table 1. Core and infall parameters towards HMCs.

\begin{tabular}{lccccc}
\hline \multicolumn{1}{c}{ HMC } & $\begin{array}{c}M_{\text {core }} \\
\left(M_{\odot}\right)\end{array}$ & $\begin{array}{c}R \\
(\mathbf{p c})\end{array}$ & $\begin{array}{c}V_{\text {infall }} \\
\left(\mathbf{k m ~ s} \mathbf{~ s}^{-1}\right)\end{array}$ & $\begin{array}{c}\dot{M}_{\text {infall }} \\
\left(M_{\odot} \mathbf{y r}^{-1}\right)\end{array}$ & Refs. $^{a}$ \\
\hline G10.62-0.38 & 82 & 0.02 & 4.5 & $3 \times 10^{-3}$ & 1,2 \\
G24.78+0.08 A1 & 130 & 0.02 & 2 & $4 \times 10^{-4}-10^{-2}$ & 2,3 \\
W51 North & 90 & 0.07 & 4 & $4 \times 10^{-2}-7 \times 10^{-2}$ & 4 \\
W51e2 & 140 & 0.01 & 3.5 & $6 \times 10^{-3}$ & 5,6 \\
G31.41+0.31 & 490 & 0.04 & 3.1 & $3 \times 10^{-3}-3 \times 10^{-2}$ & 3,7 \\
G19.61-0.23 & 415 & 0.03 & 4 & $>3 \times 10^{-3}$ & 2 \\
\hline
\end{tabular}

Notes: ${ }^{a}$ References for the core parameters: 1: Keto et al. (1988); 2: Beltrán et al. (2010); 3: Beltrán et al. (2004); 4: Zapata et al. (2008); 5: Zhang \& Ho (1997); 6: Shi et al. (2010) 7: Girart et al. (2009)

\section{Hot molecular cores}

HMCs, the cradles of $\mathrm{OB}$ stars, are dense, $n \sim 10^{7} \mathrm{~cm}^{-3}$, compact, dusty cores with temperatures in excess of $100 \mathrm{~K}$, and luminosities $>10^{4} L_{\odot}$. These cores are often found in association with typical signposts of massive star formation such as UC HII regions and maser emission of different species. As a result of the evaporation of dust grain mantles by the strong radiation of the deeply embedded early-type star, hot cores exhibit a rich chemistry observable in molecular line emission at (sub)millimeter wavelengths (e.g. Beuther 2007). In these cores, hydrogenated molecules, such as $\mathrm{NH}_{3}$, HS, oxygenbearing molecules, such as $\mathrm{SiO}, \mathrm{SO}, \mathrm{CO}$ and isotopomers, deuterated species, complex organic molecules, such as $\mathrm{CH}_{3} \mathrm{OH}, \mathrm{CH}_{3} \mathrm{CN}, \mathrm{HCOOCH}_{3}$, and pre-biotic molecules, such as $\mathrm{CH}_{2} \mathrm{OHCHO}$ (Beltrán et al. 2009), are abundant. The fact that so many different molecules are observed towards these regions allows us to estimate the physical parameters, such as temperature, density, and mass, and moreover, to study the kinematics of these young massive stellar objects, making it possible, in some cases, to distinguish infall, outflow, and rotation signatures as we will discuss next.

Infall. Despite the importance of infall to test models of massive star formation, as a result of the difficulty to detect and recognize infall, up to now there are still very few detections. A very effective method of identifying the presence of infalling gas is to observe blue-skewed profiles, also known as red-shifted self-absorption, of optically thick lines (e.g. Wu \& Evans 2003), or in case of very bright embedded continuum sources, to observe red-shifted absorption. In the latter case, the red-shifted absorption has been observed against the bright continuum emission of an embedded hypercompact HII region, as for G10.62-0.02 (Keto et al. 1988; Sollins et al. 2005) and G24.78+0.08 A1 (Beltrán et al. 2006), and in others, against the bright optically thick continuum emission from the core center, as for W51 North (Zapata et al. 2008), G19.61-0.23 (Wu et al. 2009), and G31.41+0.31 (Girart et al. 2009). As the continuum source is very bright $(\geqslant 2000 \mathrm{~K})$, it is easy to observe the colder molecular gas $(\sim 100 \mathrm{~K})$ in absorption against it. If the material surrounding the protostar(s) is not only rotating, but also accreting onto the central star(s), one expects to see absorption at positive velocities relative to the stellar velocity. This is what has been detected towards the hot cores in Table 1.

The infall rates are very high, of the order of $10^{-3}-10^{-2} M_{\odot} \mathrm{yr}^{-1}$, and in some cases, could be sufficient to quench the formation of an Hir region (Walmsley 1995), or to slow down the expansion of it. In fact, detailed models by Keto (2002) predict that trapped HC HiI regions could be long-lived, and that accretion could proceed through them in the form of ionized accretion flows.

Molecular outflows. Molecular outflows trace the earliest phases of protostellar evolution when extinction is high. They are ubiquitous phenomena in star formation, and have broadly been detected towards massive young stellar objects, from B- to O-type stars 
Table 2. List of rotating disks and toroids in high-mass (proto)stars

\begin{tabular}{clcccccc}
\hline \multirow{2}{*}{ Number $^{a}$} & \multicolumn{1}{c}{ Core } & $\begin{array}{c}M_{\text {gas }}^{\text {OH } 94} \\
\left(M_{\odot}\right)\end{array}$ & $\begin{array}{c}M_{\text {gas }}^{c} \\
\left(M_{\odot}\right)\end{array}$ & $\begin{array}{c}R \\
(\mathbf{p c})\end{array}$ & $\begin{array}{c}V_{\text {rot }} \\
\left(\mathbf{k m ~ s}^{-1}\right)\end{array}$ & Refs. $^{d}$ & $t_{\text {ff }} / t_{\text {rot }}^{e}$ \\
\hline 1 & IRAS 20126+4104 & 0.93 & 4 & 0.008 & 1.3 & 1,2 & 0.36 \\
2 & Cepheus A HW2 & 2.1 & 8 & 0.0016 & 3.0 & 3 & 0.25 \\
3 & IRAS 23151+5912 & 11 & 26 & 0.010 & 3.0 & 4 & 0.27 \\
4 & GH2O 92.67+3.07 & $12^{f}$ & 12 & 0.035 & 1.2 & 5 & 0.18 \\
5 & G29.96-0.02 & 28 & 28 & 0.011 & 1.6 & 6 & 0.10 \\
6 & G20.08-0.14N & 40 & 95 & 0.024 & 3.5 & 7 & 0.26 \\
7 & NGC 6334I & 43 & 17 & 0.0014 & 5.1 & 8,9 & 0.09 \\
8 & IRAS 18089-1732 & 59 & 45 & 0.010 & 4.0 & 10 & 0.16 \\
9 & G10.62-0.38 & 82 & 82 & 0.016 & 2.1 & 6 & 0.09 \\
10 & G28.87+0.07 & 98 & 100 & 0.029 & 0.5 & 11 & 0.03 \\
11 & G24.78+0.08 C & 98 & 250 & 0.040 & 0.5 & 12 & 0.03 \\
12 & W51 North & 160 & 90 & 0.068 & 1.5 & 13 & 0.09 \\
13 & G24.78+0.08 A2 & 163 & 80 & 0.020 & 0.75 & 12 & 0.03 \\
14 & W51e2 & 241 & 140 & 0.010 & 2.0 & 14,15 & 0.04 \\
15 & G24.78+0.08 A1 & 264 & 130 & 0.020 & 1.5 & 12 & 0.04 \\
16 & G23.01-0.41 & 274 & 380 & 0.060 & 0.6 & 11 & 0.03 \\
17 & IRAS 18566+0408 & 304 & 70 & 0.034 & 3.0 & 16 & 0.09 \\
18 & G19.61-0.23 & 415 & 415 & 0.031 & 1.0 & 6 & 0.03 \\
19 & G31.41+0.31 & 508 & 490 & 0.040 & 2.1 & 12 & 0.06 \\
20 & NGC 7538S & 607 & 100 & 0.070 & 1.35 & 17 & 0.04 \\
\hline
\end{tabular}

Notes: ${ }^{a}$ Numbers in Fig. $2 ;{ }^{b}$ Masses estimated assuming the dust opacities of Ossenkopf \& Henning (1994); ${ }^{c}$ Masses from the literature; ${ }^{d}$ References for the core parameters: 1: Cesaroni et al. (2007); 2: Cesaroni et al. (2005); 3: Patel et al. (2005); 4: Beuther et al. (2007c); 5: Bernard et al. (1999); 6: Beltrán et al. (2010); 7: Galván-Madrid et al. (2009); 8: Hunter et al. (2006); 9: Beuther et al. (2008); 10: Beuther et al. (2005); 11: Furuya et al. (2008); 12: Beltrán et al. (2004); 13: Zapata et al. (2008); 14: Zhang \& Ho (1997); 15: Shi et al. (2010); 16: Zhang et al. (2007); 17: Sandell et al. (2003); ${ }^{e}$ The free-fall times $t_{\mathrm{ff}}$ have been estimated from the masses obtained assuming the dust opacities of Ossenkopf \& Henning (1994). ${ }^{f}$ The mass of this core has been estimated from CS (Bernard et al. 1999) and is the value used to derive $t_{\mathrm{ff}}$.

with luminosities of up to $10^{6} L_{\odot}$ (Shepherd \& Churchwell 1996). Surveys of massive molecular outflows (Beuther et al. 2002; Wu et al. 2004; López-Sepulcre et al. 2009) have shown that there is a continuity in the correlations between outflow-related quantities, such as, mechanical luminosity, mechanical force, and mass loss rate, and the bolometric luminosity from low-mass to high-mass protostars. The higher the luminosity, the higher the outflow-related quantity. The fact that these correlations hold for a broad range (0.1$10^{6} L_{\odot}$ ) of luminosities suggests that the luminosity of the powering source determines the outflow energetics, and that the driving mechanism is similar for all luminosities. Note that although the possible multiplicity of massive outflows would shift the mechanical force and luminosity of the individual outflows towards lower values, the overall appearance of the correlations would remain the same (López-Sepulcre et al. 2009).

Molecular outflows may be crucial in the formation of massive stars. First of all, they can reduce the radiation pressure experienced by the gas in the infalling envelope (Krumholz et al. 2005). In fact, the radiation can escape through the cavity opened by them, which allows the infalling material to reach the central protostar. What is more, as predicted by theoretical works, outflows can be an important feedback mechanism in the process of high-mass/cluster formation (e.g. Wang et al. 2010). In fact, López-Sepulcre et al. (2010) have shown that up to $20-25 \%$ of the clump mass can be affected by molecular outflows. Molecular outflows are also important because, as shown by López-Sepulcre et al. (2010), they can help to discern whether star formation has already started in a massive core. These authors have found that for a sample of massive clumps, when the surface density $\Sigma$ is higher than $0.3 \mathrm{~g} \mathrm{~cm}^{-2}$, close to the critical theoretical value predicted by Krumholz \& McKee (2008) to form a high-mass star, the outflow detection rate is $100 \%$. And last but not least, since accretion and outflow are tightly linked, well 
collimated outflows can provide evidence for the existence of accretion disks around massive stars. In particular, the mass loss rate can provide a rough estimate of the accretion rate onto the star (e.g. Beuther et al. 2002), which is a much more difficult parameter to measure.

$\underline{\text { Rotation. }}$. In recent years an ever growing number of massive, rotating structure have been found around high-mass young stellar objects (see Table 2). Most of them have been discovered thanks to velocity gradients perpendicular to the corresponding outflow. These structures have sizes of a few thousands of AUs and masses that range from 4 to about $500 M_{\odot}$. Following Cesaroni et al. (2006), these rotating structures should be classified into two classes on the basis of the ratio between the mass of the rotating structure and that of the star. When the mass is lower than that of the central star, the object is a centrifugally supported disk. Instead, structures having masses in excess of several $10 M_{\odot}$, much greater than the mass of the central star(s), are called toroids and appear to be non-equilibrium structures. Their properties are different from those of disks around low-mass young stellar objects. These toroids may host not just a single star, but a whole cluster. Due to the fact that the gravitational potential is dominated by the massive toroid, Keplerian rotation is not possible on scales of $10^{4}$ AUs. For this reason, it has been proposed that toroids never reach equilibrium and could be transient entities with timescales of the order of the free-fall time, $\sim 10^{4} \mathrm{yr}$.

This distinction between disks and toroids has been illustrated by Beltrán et al. (2010) who have plotted $t_{f f} / t_{\text {rot }}$ versus $M_{\text {gas }}$ (re-calculated using the dust opacities of Ossenkopf \& Henning 1994) for a number of disks and toroids around high-mass (proto)stars found in the literature (see Fig. 2 and Table 2). The free-fall time $t_{f f}$ is proportional to the dynamical timescale needed to refresh the material of the toroid and $t_{\text {rot }}$ is the rotational period at the outer radius, $2 \pi R / V_{\text {rot }}$, needed to adjust the system to a new equilibrium configuration. If the structure rotates fast, the infalling material has enough time to settle into a centrifugally supported disk. Vice versa, if the structure rotates slowly, the infalling material does not have enough time to reach centrifugal equilibrium and the rotating structure is a transient toroid. Therefore, the higher the $t_{\mathrm{ff}} / t_{\mathrm{rot}}$ ratio, the more similar should be the rotating structure to a circumstellar disk. In Fig. 2, one sees that the least massive structures, which have masses comparable to or lower than that of the central star have the highest $t_{\mathrm{ff}} / t_{\text {rot }}$ ratio, while above a core mass of $\sim 10^{2} M_{\odot}$ the ratio decreases significantly.

The main conclusion, is that up to now no true disks in Keplerian rotation have been found around the most massive O-type stars, like the one imaged towards the B-type star IRAS 20126+4104 (Cesaroni et al. 2005). This could be due to an observational bias: disks could be heavily embedded inside the toroids and their emission difficult to disentangle from that of the large-scale rotating structure with current instrumentation. Another possibility could be that single disks are truncated at small radii (Bonnell \& Bate 2005) by interactions with stellar companions in the cluster (but see Ian Bonnell's contribution in this volume), or by photo-ionization (Hollenbach et al. 2000), or not detected because too distant.

Magnetic fields. A hot topic in massive star formation is the role of magnetic fields. Recent dust polarization observations of the HMCs G31.41+0.31 (Girart et al. 2009), and W51 e2 and e8 (Tang et al. 2009) have revealed magnetic field lines perpendicular to the major axis of the core, in the direction of the rotation axis, with a clear hourglass morphology. This suggests that magnetic fields play an important role in the formation of massive stars and could control the dynamical evolution of the cores (see Ramprasad Rao's contribution in this volume). What is more, Girart et al. (2009) have also found evidence of magnetic braking in the G31.41+0.31 contracting core. 


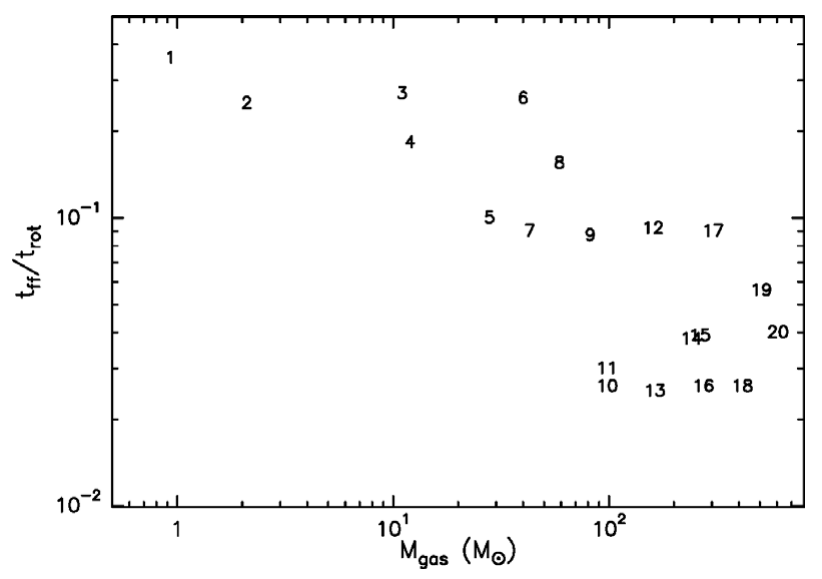

Figure 2. Free-fall timescale to rotational period ratio versus gas mass of known rotating disks and toroids. The numbers correspond to the entries of Table 2 (figure adapted from Beltrán et al. 2010). Masses estimated assuming the dust opacities of Ossenkopf \& Henning (1994).

More recently, $6.7 \mathrm{GHz}$ methanol maser polarization observations (Vlemmings et al. 2010) have determined the full 3-D magnetic field structure around Cepheus A HW2. The field is predominantly aligned along the protostellar outflow and has a strength of $23 \mathrm{mG}$. According to Vlemmings et al. (2010), the magnetic field regulates accretion onto the disk, dominates the turbulent energies by a factor of $\sim 3$, and is strong enough to stabilize the massive accretion disk and sustain the high accretion rates needed during massive star formation.

\section{Conclusions}

High-angular resolution observations of high-mass star-forming regions allow us to start studying in detail fragmentation, infall, accretion, rotation, and outflow towards these regions. However, the available data do not allow us yet to distinguish which accretionbased mechanism, monolithic accretion or competitive accretion, is the responsible for the formation of massive stars. Therefore, beside the important progress made in this field in recent years, there is still a long way to go before our understanding of the formation of all kinds of massive stars, from 8 to $\sim 100 M_{\odot}$, will be complete. In particular, although rotating structures are routinely found towards HMCs, no true accretion disk has yet been found around an O-type protostar, which could indicate that the most massive stars for via a different mechanism. In this sense, the advent of ALMA, with its superior sensitivity and resolution, will be crucial. Taking into account that the first call for ALMA early science will be by the end of this year, the search for circumstellar disks around O-type stars could end soon.

\section{References}

Bate, M. R. 2009, MNRAS, 392, 1363

Beltrán, M. T., Cesaroni, R., Codella, C., Testi, L., et al. 2006, Nature, 443, 427

Beltrán, M. T., Cesaroni, R., Neri, R., Codella, C., Furuya, R. S., et al. 2004, ApJ, 601, L187

Beltrán, M. T., Cesaroni, R., Neri, R., \& Codella, C. 2010, A\& $A$, submitted

Beltrán, M. T., Codella, C., Viti, S., Neri, R., \& Cesaroni, R. 2009, ApJ, 690, L93

Bernard, J. P., Dobashi, K., \& Momose, M. 1999, A\&A, 350, 197

Beuther, H. 2007, Proceedings of IAU S237 (Cambridge: Cambridge University Press), p. 148 
Beuther, H., Churchwell, E. B. et al. 2007, Protostars $8 \mathcal{S}$ Planets V, p. 165

Beuther, H., Schilke, P., Sridharan, T. K., Menten, K. M., et al. 2002, A\&̛A, 383, 892

Beuther, H., Walsh, A. J., Thorwirth, S., Zhang, Q., et al. 2008, A\&SA, 481, 169

Beuther, H., Zhang, Q., Hunter, T. R. et al. 2007b, A\&A, 473, 493

Beuther, H., Zhang, Q., Sridharan, T. K., \& Chen, Y. 2005, ApJ, 628, 800

Bonnell, I. A. \& Bate, M. R. 2002, MNRAS, 336, 659

Bonnell, I. A. \& Bate, M. R. 2005, MNRAS, 362, 915

Carey, S. J., Clark, F. O., Egan, M. P., Price, S. D., et al. 1998, ApJ, 508, 721

Cesaroni, R., Galli, D., Lodato, G., Walmsley, C. M., \& Zhang, Q. 2006, Nature, 444, 703

Cesaroni, R., Galli, D. et al. 2007, Protostars 85 Planets V, p. 197

Cesaroni, R., Neri, R., Olmi, L., Testi, L., et al. 2005, A\&̊A, 434, 1039

Chambers, E. T., Jackson, J. M., Rathborne, J. M., \& Simon, R. 2009, ApJS, 181, 360

Egan, M. P., Shipman, R. F., Price, S. D., Carey, S. J. et al. 1998, ApJ, 494, L199

Furuya, R. S., Cesaroni, R., Takahashi, S., Codella, C., et al. 2008, ApJ, 673, 363

Galván-Madrid, R., Keto, E., Zhang, Q., Kurtz, S, et al. 2009, ApJ, 706, 1036

Girart, J. M., Beltrán, M. T., Zhang, Q., Rao, R., \& Estalella, R. 2009, Science, 324, 1408

Hollenbach, D. J., Yorke, H. W., \& Johnstone, D. 2000, Protostars \& Planets IV, p. 401

Hunter, T. R., Brogan, C. L., Megeath, S. T., Menten, K. M., et al. 2006, ApJ, 649, 888

Jiménez-Serra, I., Caselli, P., Tan, J. C., Hernández, A. K., et al. 2010, MNRAS, in press

Keto, E. 2002, ApJ, 580, 980

Keto, E. R., Ho, P. T.P., \& Haschick, A. D. 1988, ApJ, 324, 920

Khan, F. D. 1974, A\&A, 37, 149

Krumholz, M. R., Cunningham, A. J., Klein, R. I., \& McKee, C. F. 2010, ApJ, 713, 1120

Krumholz, M. R. \& McKee, C. F. 2008, Nature, 451, 1082

Krumholz, M. R., McKee, C. F., \& Klein, R. I. 2005, ApJ, 618, L33

Krumholz, M. R., Klein, R. I. et al., 2009, Science, 323, 754

López-Sepulcre, A., Cesaroni, R., \& Walmsley, C. M. 2010, $A \mathscr{E} A$, in press

López-Sepulcre, A., Codella, C., Cesaroni, R. et al. 2009, A\&A, 499, 811

McKee, C. F. \& Tan, J. C. 2002, Nature, 416, 59

Molinari, S. \& Hi-GAL Consortium 2010, A\&A, 518, L100

Ossenkopf, V. \& Henning, Th. 1994, A\&A, 291, 943

Patel, N., Curiel, S., Sridharan, T. K., Zhang, Q., et al. 2005, Nature, 437, 109

Peretto, N., Fuller, G. A., Plume, R., Anderson, L. D., et al. 2010, A\&̈A, 518, L98

Rathborne, J. M., Jackson, J. M., Chambers, E. T., Stoijmirovic, I., et al. 2010, ApJ, 715, 322

Rathborne, J. M., Jackson, J. M., \& Simon, R. 2006, ApJ, 641, 389

Rosolowsky. E., et al. 2009 ApJS, 188, 123

Sandell, G., Wright, M., \& Forster, J. R. 2003, ApJ, 590, L45

Schuller, F. et al. 2009, A\&A A, 504, 415

Shepherd, D. S. \& Churchwell, E. 1996, ApJ, 472, 225

Shi, H., Zhao, J.-H., \& Han, J. L. 2010, ApJ, 710, 843

Sollins, P. K., Zhang, Q., Keto, E., \& Ho, P. T.P. 2005, ApJ, 624, L49

Tang, Y.-W., Ho, P. T.P., Koch, P. M., Girart, J. M., et al. 2009, ApJ, 700, 251

Vlemmings, W. H.T., Surcis, G. et al. 2010, MNRAS, 404, 134

Walmsley, M. 1995, Rev. Mex. Astron. Astrophys. Conf. Ser. 1, 137

Wang, P., Li, Z. Y., Abel, T., \& Nakamura, F. 2010, ApJ, 709, 27

Wang, Y., Zhang, Q., Pillai, T., Wyrowski, F., \& Wu, Y. 2008, ApJ, 672, L33

Wolfire, M. G. \& Cassinelli, J. P. 1987, ApJ, 319, 850

Wu, J. \& Evans, N. J. 2003, ApJ, 592, L79

Wu, Y., Qin, S.-L., Guan, X., Xue, R., et al. 2009, ApJ, 697, L116

Wu, Y., Wei, Y., Zhao, M., Shi, Y., et al. 2004, A\& $A$, 426, 503

Zapata, L. A., Palau, A., Ho, P.T P., Schilke, P., et al. 2008, A\&A, 479, L25

Zhang, Q. \& Ho, P. T. P. 1997, ApJ, 488, 241

Zhang, Q., Sridharan, T. K., Hunter, T. R., Chen, Y., et al. 2007, A\&\&A, 470, 269

Zhang, Q., Wang, Y., Pillai, T., \& Rathborne, J. 2009, ApJ, 696, 268 